9 Vu DH, Alffenaar JW, Edelbroek PM, et al. Dried blood spots: a new tool for tuberculosis treatment optimization. Curr Pharm Des 2011; 17: 2931-2939.

10 Esposito S, Codecasa LR, Centis R. The role of therapeutic drug monitoring in individualised drug dosage and exposure measurement in tuberculosis and HIV co-infection. Eur Respir J 2015; 45: 571-574.

\title{
B-blockers in pulmonary arterial hypertension: generation might matter
}

To the Editor:

We read with great interest the article from BANDYOPADHYAY et al. [1] regarding the outcomes of $\beta$-blockers use in pulmonary arterial hypertension $(\mathrm{PAH})$. This study adds another piece in the very controversial debate opposing pro- and anti-supporters of $\beta$-blockers use in PAH. In this study, patients had idiopathic PAH (IPAH) or connective tissue disease-associated PAH. PAH patients receiving $\beta$-blockers had a similar survival and time to clinical worsening events compared with patients not receiving them. Functional outcomes were similar, although $\beta$-blocker use was associated with a tendency towards shorter walking distance.

The most recent guidelines in PAH do not recommend the use of $\beta$-blockers, unless recommended by comorbidities, because no convincing data are available on their usefulness and safety $[2,3]$. Although the recent study by BANDYOPADHYAY et al. [1] appears like the last demonstration of $\beta$-blocker inefficiency in the setting of PAH, we rather see it as DE MAN and HANDoko [4] pointed out in their recent editorial, as an emerging signal that the dangers of $\beta$-blockers in $\mathrm{PAH}$ patients are smaller than previously thought. We would also point out that $\beta$-blockers are a wide and heterogeneous group of molecules. $\beta$-blockers differ in terms of $\beta$-adrenergic receptors selectivity, adjunctive effects on $\alpha$-receptors and effects on oxidative stress and inflammation [5]. The "first-generation" compounds, such as propranolol and nadolol, are non-selective agents with equal affinities for blocking $\beta 1$ and $\beta 2$ receptors and no important pharmacological properties other than $\beta$-adrenergic blockade. In addition, the $\beta 2$-subtype is the predominant adrenergic receptor present in the pulmonary vasculature. Blockade of the $\beta 2$-receptors may lead to smooth muscle contraction, which could result in an increase in pulmonary vascular resistance and right ventricle pressure [6]. The "second-generation" of $\beta$-blockers, such as metoprolol or bisoprolol, are $\beta 1$-selective (cardioselective) and lack $\beta 2$-receptor blockade, therefore reducing some of the peripheral and pulmonary side effects. The "third generation" has vasodilating properties in addition to its $\beta$-blockade action. This specific generation has different pharmacological profiles as nebivolol is highly $\beta 1$-selective with vasodilating activity possibly due to an interaction with the L-arginine/nitric oxide pathway while carvedilol blocks $\beta 1, \beta 2$ and $\alpha 1$ adrenergic receptors, the latter possibly acting as potent vasodilator [7]. Hence, the third generation might have the advantage of right heart afterload reduction to counteract the negative inotropic properties of adrenergic withdrawal, which raise interest for this specific generation of molecules as an add-on to current therapies. However, prospective clinical trials must be performed to clarify whether or not targeting the $\beta$-adrenergic system can be added to the current treatment paradigm of PAH [8].

The initial belief that $\beta$-blockers may be harmful in PAH was strengthened by a small study in 10 patients in the setting of porto-pulmonary hypertension, in whom withdrawal of propranolol (eight out of 10) or atenolol (two out of 10) treatment was associated with an improved 6-min walk distance [9] and further strengthened by a case report in which the addition of a single dose of $25 \mathrm{mg}$ oral metoprolol combined with amiodarone caused a near-fatal acute cardiac decompensation [10]. However, this is not surprising since administration of first-generation compounds, such as propranolol, causes a decrease in contractile state. This, plus a concomitant increase in systemic vascular resistance from $\beta 2$-receptor blockade, leads to a profound decrease in cardiac output, which results in a drug intolerance rate of $20 \%$ in dilated cardiomyopathy [7]. Alternatively, no obvious explanation for the $\beta$-blockers and amiodarone interaction exists. An additive effect in patients could be considered as amiodarone has also $\beta$-adrenergic blockade effect [11].

A recent retrospective study dealing mainly with the second-generation of $\beta$-Bs bisoprolol reported that their use was associated with a reduction in right ventricle (RV) dilatation and an improvement of tricuspid annular plane systolic excursion, which was attributed to a reduction of RV fibrosis [12]. However, in two recent retrospective studies, clinical, functional and hemodynamic outcomes of PAH patients were analysed coincidentally taking $\beta$-blockers, e.g. for treatment of arrhythmias. In these studies, second-generation 
$\beta$-blockers (mainly metoprolol) did not appear to exert detrimental effects in PAH patients even if beneficial effects of $\beta$-blockers were not specifically studied $[13,14]$. Thus, discrepancies remain in the current literature regarding the second-generation of $\beta$-blockers effects and benefits; although detrimental effects seem not to be present as it was with the use of the first generation $\beta$-blockers.

Recent focuses have shed light on the third generation of $\beta$-blockers in PAH. We recently published that nebivolol normalised the pathological phenotype of pulmonary endothelial cells harvested from $\mathrm{PAH}$ patients and alleviated pulmonary hypertension in rats exposed to monocrotaline [15], opening new concrete therapeutic targets in $\mathrm{PAH}$ that could have positive impacts on pulmonary vasculature, neurohormonal axis and right heart failure all at once. Moreover, in a single-arm open-label pilot study (registered at www.clinicaltrials.gov with identifier NCT00964678) designed to evaluate the feasibility and safety of add-on treatment with carvedilol in six patients with stable PAH and RV dysfunction (functional classes II and III), the initial data suggested that treatment with carvedilol in patients with PAH receiving standard vasodilator drug therapy is feasible and safe, and leads to significant improvement in RV ejection fraction and stroke volume, without changes in left ventricle ejection fraction. There was also a trend toward a 6-min walk distance increase [16]. Finally, therapy with nebivolol led to a significant functional improvement in 12 patients with idiopathic $\mathrm{PAH}$ and reductions in RV dimensions and blood endothelin-1 levels [17].

In conclusion, different clinical, functional and haemodynamic responses exist between the different generations of $\beta$-blockers in $\mathrm{PAH}$. The pooling of $\mathrm{PAH}$ patients receiving different generations of $\beta$-blockers raised the question whether or not similar clinical, functional and haemodynamic results would have been observed if patients were stratified according to generations. Of course, the present retrospective cohort of the study by Bandyopadhyay might not allow stratification. Therefore, we believe that adequately powered prospective studies are now needed in light of the actual possible benefits and lack of detrimental effects of second- and third-generation $\beta$-blockers in $\mathrm{PAH}$.

- @ERSpublications

Malenfant and Perros discuss the potential usefulness of third generation $\beta$-blockers in pulmonary hypertension http://ow.ly/VKMFn

Simon Malenfant ${ }^{1}$ and Frédéric Perros ${ }^{1,2}$

${ }^{1}$ Pulmonary Hypertension and Vascular Biology Research Group, Centre de recherche de l'Institut Universitaire de cardiologie et de pneumologie de Québec, Université Laval, Québec, QC, Canada. ${ }^{2}$ UMR_S 999, Univ. Paris-Sud; INSERM; Laboratoire d'Excellence (LabEx) en Recherche sur le Médicament et l'Innovation Thérapeutique (LERMIT), Centre Chirurgical Marie Lannelongue, Le Plessis Robinson, France.

Correspondence: F. Perros, Centre de recherche de l'Institut Universitaire de cardiologie et de pneumologie de Québec, Service de Pneumologie, 2725, chemin Sainte-Foy, Québec, QC, G1V 4G5, Canada.

E-mail: frederic.perros@criucpq.ulaval.ca

Received: July 282015 | Accepted after revision: Sept 032015

Conflict of interest: None declared.

\section{References}

1 Bandyopadhyay D, Bajaj NS, Zein J, et al. Outcomes of $\beta$-blocker use in pulmonary arterial hypertension: a propensity-matched analysis. Eur Respir J 2015; 46: 750-760.

2 Galiè N, Humbert M, Vachiery J-L, et al. 2015 ESC/ERS Guidelines for the diagnosis and treatment of pulmonary hypertension. Eur Respir J 2015; 46: 903-975

3 Galiè N, Humbert M, Vachiery J-L, et al. 2015 ESC/ERS Guidelines for the diagnosis and treatment of pulmonary hypertension. Eur Heart J 2015; [In press DOI: 10.1093/eurheartj/ehv317]

4 de Man FS, Handoko ML. $\beta$-blockers in pulmonary arterial hypertension: evolving concepts of right heart failure. Eur Respir J 2015; 46: 619-621.

5 Barrese V, Taglialatela M. New advances in $\beta$-blocker therapy in heart failure. Front Physiol 2013; 4: 323.

6 Leblais V, Delannoy E, Fresquet F, et al. $\beta$-adrenergic relaxation in pulmonary arteries: preservation of the endothelial nitric oxide-dependent $\beta 2$ component in pulmonary hypertension. Cardiovasc Res 2008; 77: 202-210.

7 Bristow MR. beta-adrenergic receptor blockade in chronic heart failure. Circulation 2000; 101: 558-569.

8 Rubin LJ. The Beta-Adrenergic Receptor in Pulmonary Arterial Hypertension: A Novel Therapeutic Target?. J Am Coll Cardiol 2015; 65: 681-683.

9 Provencher S, Herve P, Jais X, et al. Deleterious effects of beta-blockers on exercise capacity and hemodynamics in patients with portopulmonary hypertension. Gastroenterology 2006; 130: 120-126.

10 Peacock A, Ross K. Pulmonary hypertension: a contraindication to the use of $\beta$-adrenoceptor blocking agents. Thorax 2010; 65: 454-455.

11 Boutitie F, Boissel JP, Connolly SJ, et al. Amiodarone interaction with $\beta$-blockers: analysis of the merged EMIAT (European Myocardial Infarct Amiodarone Trial) and CAMIAT (Canadian Amiodarone Myocardial Infarction Trial) databases. The EMIAT and CAMIAT Investigators. Circulation 1999; 99: 2268-2275.

12 Moretti C, Grosso Marra W, D’Ascenzo F, et al. $\beta$ blocker for patients with pulmonary arterial hypertension: A single center experience. Int J Cardiol 2015; 184: 528-532. 
13 So PP-S, Davies RA, Chandy G, et al. Usefulness of $\beta$-blocker therapy and outcomes in patients with pulmonary arterial hypertension. Am J Cardiol 2012; 109: 1504-1509.

14 Thenappan T, Roy SS, Duval S, et al. $\beta$-blocker therapy is not associated with adverse outcomes in patients with pulmonary arterial hypertension: a propensity score analysis. Circ Heart Fail 2014; 7: 903-910.

15 Perros F, Ranchoux B, Izikki M, et al. Nebivolol for improving endothelial dysfunction, pulmonary vascular remodeling, and right heart function in pulmonary hypertension. J Am Coll Cardiol 2015; 65: 668-680.

16 Grinnan D, Bogaard H-J, Grizzard J, et al. Treatment of group I pulmonary arterial hypertension with carvedilol is safe. Am J Respir Crit Care Med 2014; 189: 1562-1564.

17 Martyniuk TV, Konosova ID, Chazova IE. [Use of nebivolol in patients with idiopathic pulmonary hypertension: results of the pilot study.] Ter Arkh 2012; 84: 49-53.

\section{Cameroon's MDR-TB treatment programme jeopardised by cross-border migration}

To the Editor:

We read with interest in a recent issue of the European Respiratory Journal, a paper by LÖNNROTH et al. [1] entitled. "Towards tuberculosis elimination: an action framework for low-incidence countries". The authors highlight cross-border migration as a major challenge for tuberculosis (TB) control efforts and their action framework pleads for strong international partnerships for reaching global TB elimination targets. To further comment on the challenges posed by cross-border migration, we here report on a recent experience of Cameroon's National TB Programme (NTP).

Since 2005, Cameroon's NTP has been treating multidrug-resistant (MDR)-TB patients with standardised therapeutic regimens inspired by the experiences of VAN DEUN and colleagues [2,3] in Bangladesh. From 2008 to 2012, patients were treated using a short duration regimen (12 months); a cohort assessment of the first 150 patients revealed an $89 \%$ therapeutic success rate [4]. Since 2013, patients have been treated using a 9-month regimen (4KmMfxPtoClzEZH/5MfxClzEZ; known as the "Bangladesh regimen"). Evaluation of a first cohort shows a therapeutic success rate of $82 \%$. In 2014, a total of 91 patients were put on treatment. Unlike the World Health Organization (WHO) recommended regimens for the management of drug-resistant $\mathrm{TB}$, short-course regimens are easily implemented, use less toxic drugs, are more tolerable, limit the use of injectables and reduce the risk of treatment interruption and defaulting [5, 6]. Moreover, the fact that patients in Cameroon have very little or no exposure to second-line anti-TB drugs, particularly to fluoroquinolones, for the treatment of diseases other than TB, might contribute to the programme success. How fluoroquinolone resistance in MDR-TB patients compromises favourable treatment outcomes was shown by FALzON et al. [7] in a large individual patient data meta-analysis.

For several years, MDR-TB patients from neighbouring countries not offering MDR-TB treatment, for example Equatorial Guinea, have been put on standardised treatment in Cameroon, based on the assumption that the epidemiological situation in these countries may possibly be similar to that of Cameroon. Since the beginning of 2015, Cameroon's NTP has seen a large influx of MDR-TB patients from Equatorial Guinea. 14 MDR-TB patients have been put on treatment on the basis of an Xpert MTB/RIF (Cepheid, Maurens-Scopont, France) test result "Rifampicin-resistance" in accordance with the national guidelines.

An early patient from Equatorial Guinea proved resistant to fluoroquinolones in late 2013 and eventually failed her treatment, and was considered as an "anecdotal" event. Recently, a second patient's cultures did not convert to negative after 6 months of treatment. Drug-resistance testing by line probe assay (LPA) once again brought to light resistance to fluoroquinolones. Interviews with patients revealed that most of them had been exposed to repetitive courses of ciprofloxacin (Cfx) for the treatment of typhoid fever, a pathology widely over-diagnosed and over-treated in Central Africa. Table 1 summarises epidemiological and clinical characteristics of the Equatorial Guinean patients put on treatment since 2013 with LPA results awaited for some of them.

Two practical questions arise immediately. Should the 9-month regimen of patients with a history of repetitive exposure to Cfx who are presently under treatment be changed and how? Also, how best could patients who do not convert be treated and where to perform the treatment? 